\title{
Modulation of a Photoswitchable Dual-Color Quantum Dot containing a Photochromic FRET Acceptor and an Internal Standard
}

\author{
Sebastián A. Díaz, ${ }^{\dagger \neq}$ Luciana Giordano, ${ }^{\ddagger}$ Thomas M. Jovin, ${ }^{*} \neq$ and Elizabeth A. Jares-Erijman ${ }^{\dagger}$ \\ †Departamento de Química Orgánica, Facultad de Ciencias Exactas y Naturales, Universidad de Buenos Aires, CIHIDECAR, \\ CONICET, 1428 Buenos Aires, Argentina \\ ${ }^{\ddagger}$ Laboratory of Cellular Dynamics, Max Planck Institute for Biophysical Chemistry, Am Fassberg 11, 37077 Göttingen, Germany
}

\section{Supporting Information}

ABSTRACT: Photoswitchable semiconductor nanoparticles, quantum dots (QDs), couple the advantages of conventional QDs with the ability to reversibly modulate the QD emission, thereby improving signal detection by rejection of background signals. Using a simple coating methodology with polymers incorporating a diheteroarylethene photochromic FRET acceptor as well as a spectrally distinct organic fluorophore, photoswitchable QDs were prepared that are small, biocompatible, and feature ratiometric dual emission. With pro-

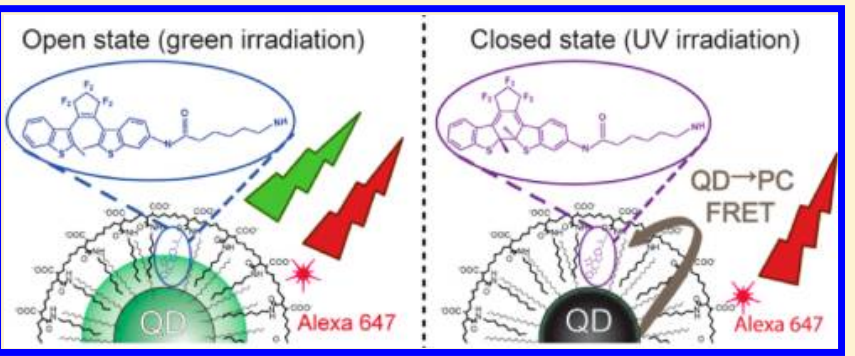
grammed irradiation, the fluorescence intensity ratio can be modified by up to $\sim 100 \%$.

KEYWORDS: Quantum dots, FRET, photochromism, internal standard, ratiometric imaging

ince their inception semiconductor nanocrystals, or $\checkmark$ quantum dots (QDs), have been applied widely in biological imaging, ${ }^{1}$ most recently for multiplexed analysis, ${ }^{2}$ single particle imaging, ${ }^{3}$ prolonged real-time visualization, ${ }^{4}$ and super resolution microscopy. ${ }^{5}$ QDs are particularly well suited to these tasks due to their broad excitation, narrow emission, photostability, and brightness. Despite their many virtues, colloidal QDs are generally synthesized in organic solvents and require surface modification in order to achieve solubility in aqueous media and biocompatibility. These aims are met either by replacing or by capping the original hydrophobic surface ligands, ${ }^{6}$ techniques that also provide the main route to further functionalization of the QDs for targeting and multiparametric biosensing. ${ }^{7,8}$

Förster resonance energy transfer (FRET) is an efficient technique for the modulation of $\mathrm{QD}$ fluorescence. ${ }^{9-12}$ Photochromic compounds (PCs) with two states differing in absorption properties have been introduced as FRET acceptors in a configuration we have previously termed pcFRET. ${ }^{13-15}$ The system constitutes a reversible switch based on FRET under control by light. A very suitable family of photochromic compounds for pcFRET comprises the thiophene-based diheteroarylethenes, ${ }^{16}$ introduced by Irie, ${ }^{17,18}$ which have two thermally stable states (open form, oPC, and photocyclized closed form, cPC) that can undergo numerous photocycles without fatigue. The open form can assume antiparallel and parallel conformations of which only the former can undergo photocyclization. Several water-soluble diheteroarylethene PCs have been designed, ${ }^{19,20}$ but they still require a hydrophobic microenvironment for optimum photoconversion. ${ }^{21}$
We have previously reported ${ }^{22}$ the design of photoswitchable QDs (psQDs) based on the strategy introduced by the Parak laboratory ${ }^{23}$ for solubilizing organic nanoparticles with coats of amphiphilic comb-polymers. [In referring to the previously reported psQD construct we will use the term Gen-1]. Such polymer-capped QDs have been targeted to biological applications in aqueous media. ${ }^{24,25}$ The amphiphilic polymer can be functionalized both prior to or after nanoparticle coating $^{7}$ with various groups located either in the hydrophobic interior or hydrophilic exterior of the coating polymer. In our application, the wrapped QDs architecture permits placement of the PC molecules in the hydrophobic compartment demarcated by the outer surface of the semiconductor QD and the polymer cap. ${ }^{22}$

We report here a new structural design that creates a biocompatible photoswitchable QD with a secondary dye (Alexa Fluor647 cadaverine) acting as an internal standard and thus allowing dual-color, ratiometric sensing and imaging (Scheme 1). The psQD is smaller than other related constructs $^{26-30}(\sim 5 \mathrm{~nm}$ diameter by EM, a $\sim 14 \mathrm{~nm}$ hydrodynamic diameter; see Supporting Information), does not exhibit fatigue, and is readily photomodulatable, such that the emission can be externally controlled. The ratiometric signal changes by $\sim 100 \%$ and allows sensitive, selective detection by suppression of background and photobleaching contributions using phase-sensitive techniques. These can be applied in the steady-state ${ }^{31}$ as well as in the nanoseconds

Received: $\quad$ March 21, 2012

Revised: $\quad$ May 15, 2012 
Scheme 1. Dual-Color psQD Schematic ${ }^{a}$

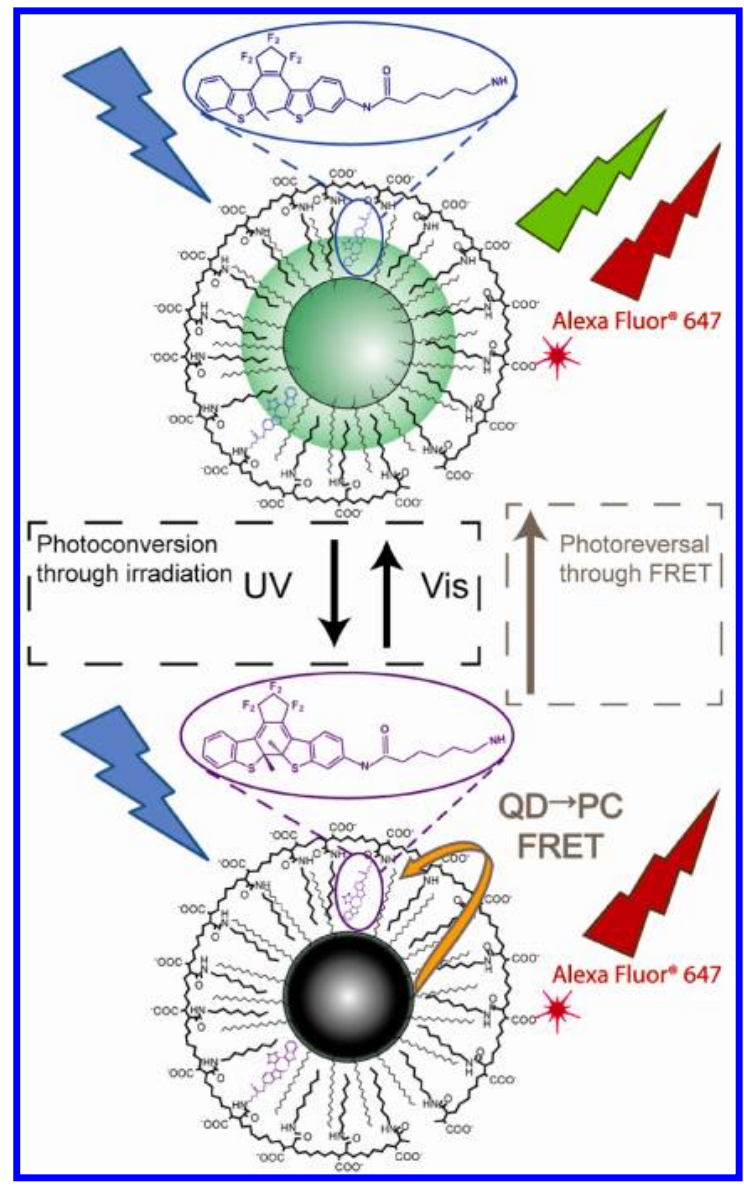

${ }^{a}$ The fluorescence of the QD is modulated by the photoconversion of the PC while the Alexa647 fluorescence is constant. The PC in the open form (oPC) is photoconverted with UV irradiation to the closed form ( $\mathrm{cPC}$ ), which can then be photoreversed by direct excitation with visible light, or via FRET from the $\mathrm{QD}$ acting as a donor.

regime, ${ }^{32}$ in the latter case exploiting the different $\mathrm{QD}$ and Alexa647 fluorescence decay kinetics.

The preparation of the dual-color psQDs begins with the synthesis of the Alexa doped photochromic amphiphilic polymer (Scheme 2). Certain modifications of the methodology previously reported for dye-doped poly(maleic anhydride) based polymers ${ }^{22,33,34}$ were required for introducing the double functionalization.

For the polymer synthesis $6 \mathrm{mg}(1 \mu$ mole polymer, 40 $\mu$ moles monomer) of PMA (Sigma-531278, MW 6000) was introduced into a dry $10 \mathrm{~mL}$ flask. Two milliliters of a DMF:DMSO solution of Alexa647 cadaverine (Invitrogen, A30679; $0.16 \mu$ moles, or 4 molecules per 1000 maleic anhydride monomers) were added. The solution was stirred at $60^{\circ} \mathrm{C}$ for $90 \mathrm{~min}$ and then $7.7 \mathrm{mg}$ (14 $\mu$ moles) of PCahx [6-amino- $\mathrm{N}$-(3(3,3,4,4,5,5-hexafluoro-2-(2-methylbenzo[b]thiophen-3-yl)cyclopent-1-enyl)-2-methylbenzo[b]thiophen-6-yl)hexanamide; for synthetic details of PCahx see Supporting Information] was added in the minimal possible volume of tetrahydrofuran. The PCahx is a diheteroarylethene PC with a 6 carbon linker terminating in a primary amine. This linker positions the PC closer to the QD than in the Gen-1 psQD as well as increasing the yield of conjugation to the polymer. From prior experience with PCahx, we anticipated a conjugation yield of $\sim 20 \%$, corresponding to $\sim 3$ PC groups/polymer. We simplify the
Scheme 2. Spectral Signature of Dual-Color psQD Components and Chemical Architecture of Conjugated Polymer $^{a}$

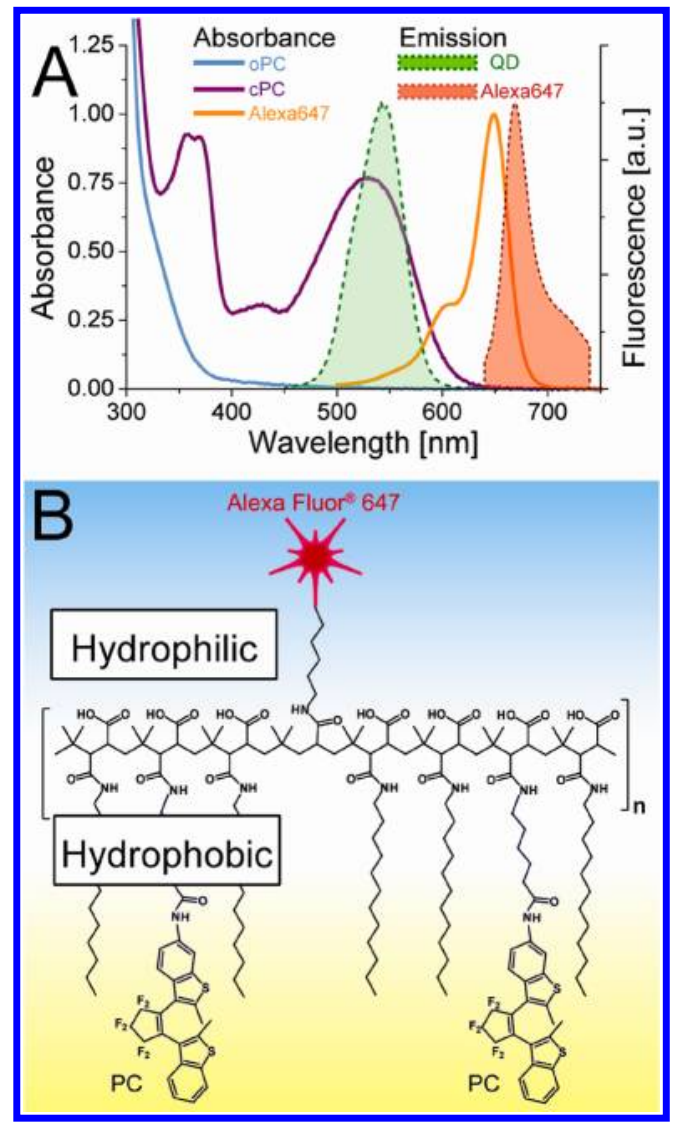

${ }^{a}$ (A) Superposition of absorbance (solid lines) and emission (filled areas) spectra of PC, QD, and Alexa647, demonstrating the PC spectral overlap with the QD but not with the Alexa647. The spectra are normalized by their peak values. (B) Schematic of photochromic amphiphilic comb polymer doped with Alexa647.

terminology by henceforth referring to PCahx as PC. Dodecylamine $(1.85 \mathrm{mg}, 10 \mu$ moles $)$ was added to the flask and the reaction was left overnight at $60{ }^{\circ} \mathrm{C}$. An additional 3.7 $\mathrm{mg}(20 \mu$ moles $)$ of dodecylamine was added and allowed to react for $4 \mathrm{~h}$. The product was purified and characterized as previously described. ${ }^{22}$ The nomenclature adopted for the polymer product represents modifications to the polyisobutylene-alt-maleic anhydride backbone as percentages of anhydride rings coupled to the added molecules. Thus, the final polymer is denoted PMA 7PC 75C12 0.3Al; for every 1000 original maleic anhydride monomers there are 750 dodecylamine chains, 70 PC molecules, and 3 Alexa647.

We utilized Series A CSS $540 \mathrm{~nm}$ QDs CdSe/CdS/ZnS core-shell-shell nanoparticles ${ }^{35}$ (CAN GmbH, Hamburg) emitting at $540 \mathrm{~nm}$ and rendered soluble in organic solvents by lipophilic surface ligands (TOPO/TOP/HDA). The coated QDs were transferred to an aqueous medium and purified following a simple, previously reported methodology. ${ }^{22}$ The polymer self-assembly is directed by interdigitations of the aliphatic chains with the lipophilic ligands on the QD surface. The PC is preferentially localized to the hydrophobic microenvironment established by the QD surface ligands and the dodecylamine chains of the polymer. The hydrophilic Alexa647 and pendant carboxylic groups are presumed to 
extend into the aqueous medium, conferring a negative surface charge and solubility to the nanoparticle. The final construct is denoted a dual-color psQD (dual-color photoswitchable quantum dot). The properties and data presented below were determined in $50 \mathrm{mM}$ Na-borate buffer, $\mathrm{pH}$ 9.0, under aerobic conditions. Stability was maintained in the $\mathrm{pH}$ range 7-12 and no adverse effects were perceived upon lowering the salt concentration to $0.5 \mathrm{mM}$. The properties of the dual-color psQDs were unaltered after storage for 6 months.

The absorption and fluorescence spectra at $20{ }^{\circ} \mathrm{C}$ of dualcolor psQDs were acquired on a Cary 100 UV-vis Spectrophotometer and a Cary Eclipse Fluorimeter (Varian), respectively. The concentrations of the nanoparticle solutions were in the range of $0.1-0.5 \mu \mathrm{M}$. For fluorescence measurements, the maximum absorbance of the solution was maintained at $<0.1$ to avoid internal filter effects and trivial reabsorption, and low levels of excitation light were utilized for monitoring the system so as to avoid photoconversion of the PCs. The phase transfer shifted the peak of the QD emission to $550 \mathrm{~nm}$ and led to a reduction of the quantum yield to 0.26 , a value similar to that reported for other polymer-coated QDs. ${ }^{36}$

The photochrome composition of the dual-color psQD was determined by resolving the absorbance spectrum into its constituent contributions. The $A_{525}$ of the dual-color psQDs with all the $\mathrm{PC}$ in the open form was assumed to originate exclusively from the semiconductor, thus permitting an estimation of the nanoparticle concentration. The increment in $A_{540}$ upon UV irradiation was assigned to changes in the relative concentrations of $\mathrm{oPC}$ and $\mathrm{cPC}$. The concentrations of CPC, and thus of total PC and number of PCs per QD, were obtained from the known differences in extinction coefficients and the $\mathrm{CPC}$ fraction in the photostationary state $\left(\alpha_{\mathrm{PS}}\right.$, see below). The $A_{650}$ originated solely from the Alexa647 and thus an Alexa647/QD ratio could also be computed. In interpreting the data, we assumed random distributions of the PC and reference probes on the polymer and of the polymer on the QD. The calculations yielded mean values of $1.2 \pm 0.1$ Alexa647 and $35 \pm 1 \mathrm{PC}$ per QD, corresponding to $\sim 400-500$ monomers of polymer (or $\sim 10-13$ polymers) per QD. The polymer content was one-half that of the Gen-1 psQD. The more efficient packing was reflected in the ease of QD coating and phase transfer. From the low value for Alexa647, the fractional distributions for QDs with $0,1,2$ and $>2$ probes would be $0.30,0.36,0.22$, and 0.12 , respectively.

Scheme 2A shows the most pertinent spectral properties of the individual components of the dual-color psQD. The latter exhibited two emission wavelengths, 550 and $666 \mathrm{~nm}$, corresponding to the $\mathrm{QD}$ and Alexa647, respectively. Both emissions could be elicited over a broad excitation band corresponding to the QD. As indicated above, QDs can act as FRET donors, ${ }^{10,37-39}$ in this case not only to the PC groups but also to the Alexa647, which will thus exhibit emission upon excitation throughout the QD excitation band. The computed Förster transfer parameter ${ }^{13}\left(R_{\mathrm{o}}\right)$, the donor-acceptor distance for which the energy transfer efficiency is 0.5 , are the following: QD-oPC, $1.3 \mathrm{~nm}$; QD-cPC, $4.1 \mathrm{~nm}$; QD-Alexa647: $4.1 \mathrm{~nm}$, assuming two-thirds for the value of orientation factor $\kappa^{2}$. The similar values for QD-cPC and the QD-Alexa647 result from compensation of the lesser spectral overlap in the latter case by the much larger extinction coefficient of Alexa647, resulting in a similar overlap integral $(J) .^{13}$

We define an emission ratio (ER) as the Alexa647 emission at $666 \mathrm{~nm}$ divided by the QD emission at $550 \mathrm{~nm}$. Alexa647 can be excited selectively from 580 to $660 \mathrm{~nm}$. Thus, the ER is adjustable by selection of one or more excitation band(s). In the experiments described below, the selected excitation wavelengths of 400 and $600 \mathrm{~nm}$ were optimal in that they correspond to minima in the PC absorbance.

Reversible photoswitching of the PC probe in the dual-color psQD was achieved by alternating irradiation with UV light $\left(340 \pm 10 \mathrm{~nm}, 1.1 \mathrm{~mW} \mathrm{~cm}^{-2}\right)$ and visible/green light (545 \pm $\left.10 \mathrm{~nm}, 6.2 \mathrm{~mW} \mathrm{~cm}^{-2}\right)$. Sequential pulses $(\sim 10 \mathrm{~s})$ of either UV or visible light were utilized to achieve the two photostationary states, ${ }^{14,22}$ the first consisting exclusively of oPC, and the second of a distribution between oPC and $\mathrm{CPC}$ established by UV irradiation. Further references to the photostationary state assume the latter condition. The changes in the absorbance and fluorescence spectra of the dual-color psQD upon passing from the open to the closed states are shown in Figure 1. The

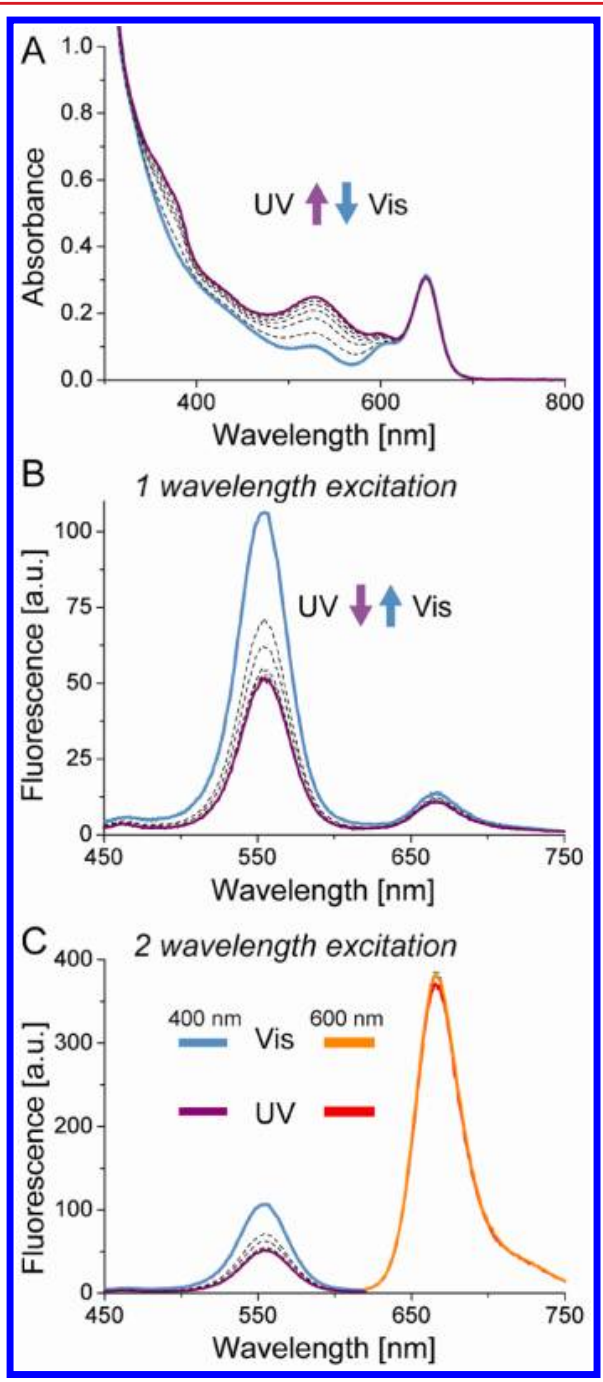

Figure 1. Spectroscopic monitoring of dual-color psQD. Irradiation consisted of consecutive $10 \mathrm{~s}$ pulses of either UV or visible light until no further change was observed; UV, $340 \pm 10 \mathrm{~nm}$ (irradiance $1.1 \mathrm{~mW}$ $\mathrm{cm}^{-2}$ ) and vis, $545 \pm 10 \mathrm{~nm}$ (irradiance $6.2 \mathrm{~mW} \mathrm{~cm}^{-2}$ ). Temperature, $20{ }^{\circ} \mathrm{C}$. (A) Absorbance spectra (B) Fluorescence spectra of dual-color psQD with excitation at $400 \mathrm{~nm}$. (C) Fluorescence spectra of dualcolor psQD with excitation wavelength at $400 \mathrm{~nm}$ for QD detection and $600 \mathrm{~nm}$ for Alexa647 detection. The $600 \mathrm{~nm}$ excitations are represented by two colors (orange, red); the signals differed very slightly. 
photostationary state of PC conjugated to the polymer corresponds to a $22 \%$ conversion $\left(\alpha_{\mathrm{PS}}=0.22\right)$ of the open to the closed states (for calculations and details see Supporting Information $1 \mathrm{~B}$ ). The photoconversion process of the dualcolor psQD is more complex due to the photoreversion caused by FRET from the QD to the CPC and the existence of two subpopulations differing in FRET efficiency (see below). Thus, the photostationary state corresponds to a lower $\alpha_{\mathrm{PS}}$ than in the case of the free or polymer-conjugated PC (see calculations and discussion below).

The maximum observed quenching of fluorescence at the $550 \mathrm{~nm}$ peak upon exposure to UV light was $52 \%$ (mean $48 \pm$ $2 \%)$. Irradiation with visible light restored the fluorescence to the initial state. Using a single excitation wavelength $(400 \mathrm{~nm})$, the ER changed from 0.13 to 0.22 (66\% increase). By exciting both probes individually (QD at $400 \mathrm{~nm}$, Alexa647 at $600 \mathrm{~nm}$ ), the ER changed from 4.0 to 8.0 (100\% increase). The difference in ER values is due to the correlated decreases in both the QD and Alexa647 emission in the single excitation mode. Quenching of the QD by the cPC also reduces FRET to the Alexa647 because of the additional competing deactivation pathways of the QD (Figure 1B). When excited directly, the Alexa647 standard was unaffected by the photoconversion of the PC, resulting in a considerably larger ER change. A slight photobleaching was evident upon prolonged irradiation.

The dual-color psQD were cycled through the open-closed states by alternating lengthy (180 s) exposures to UV or visible light and monitoring the QD and Alexa647 emissions (Figure 2). The first irradiation with UV led to a significant decrease in

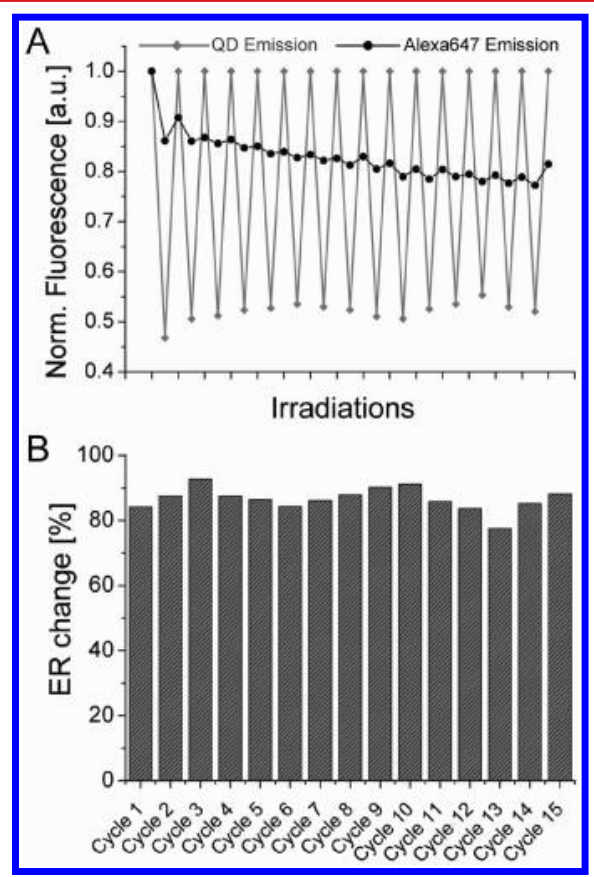

Figure 2. Spectroscopic monitoring of dual-color psQD cycled through open-closed states. Irradiation was for a continuous $180 \mathrm{~s}$; $340 \pm 10 \mathrm{~nm}$ (irradiance $1.1 \mathrm{~mW} \mathrm{~cm}^{-2}$ ) and $545 \pm 10 \mathrm{~nm}$ (irradiance $6.2 \mathrm{~mW} \mathrm{~cm}^{-2}$ ). Temperature, $20{ }^{\circ} \mathrm{C}$. (A) Emission of QD after each irradiation with excitation at $400 \mathrm{~nm}$ and detection at $550 \mathrm{~nm}$. Emission of Alexa647 after each irradiation with excitation at $600 \mathrm{~nm}$ and detection at $666 \mathrm{~nm}$. (B) Change in the ER during each cycle upon transition from the open to the closed state.

the Alexa647 signal while subsequent cycles produced a more moderate decrease indicative of photobleaching (Figure 2A).
The latter effect could be minimized by eliminating oxygen from the solution with scavengers. ${ }^{40} \mathrm{We}$ attribute the initial step to redistribution of the Alexa647 from the more viscous interior to the exterior of the polymer coat, lowering the quantum yield due to facilitation of bond rotation. ${ }^{40}$ The hypothesis was supported by an observed decrease in FRET from the QD to the Alexa647 (data not shown). The slight reversible steps in Alexa647 emission were independent of the state of the PC since they were observed when irradiating repeatedly at any given wavelength.

The QD did not exhibit photobleaching but some photobrightening was evident (compensated in Figure 2A by normalization). Photobrightening is an inherent property of many QDs ${ }^{41,42}$ and we observed the phenomenon in naked QDs as well as polymer-coated QDs not functionalized by dyes. The influence of this phenomenon can be minimized by prior irradiation. ${ }^{43}$ The photomodulation of QD fluorescence was robust over the 15 measured cycles in which the ER changed by $87 \pm 4 \%$ (Figure 2B). Neither fatigue due to the photodegradation of the PC nor spontaneous thermal reversal of $\mathrm{CPC}$ was evident at room temperature. Thus, the state of the dualcolor psQD was controlled solely via the selected mode of irradiation.

The dual-color psQD constitutes an inherently complex photophysical system by virtue of incorporating a QD donor and two distinct families of acceptors, one of which (the PC) undergoes photoinduced chemical transformations. As in the case of the Gen-1 psQDs, ${ }^{22}$ the intermediate as well as the photostationary states of the PC groups generated with pulses of irradiation are heterogeneous in terms of the open/closed form distribution. It was necessary to again invoke the existence of two distinct acceptor populations differing in PC number and FRET efficiency as the simplest means for accommodating the combined absorbance and fluorescence transition data (Figure 1A,B; Supporting Information).

The original photophysical model ${ }^{22}$ has been improved in the ensuing treatment, both in details of the formalism and the nature and number of parameters fit to the data. ${ }^{22}$ We define two classes $(1,2)$ of cPC FRET acceptors, class 1 arbitrarily being more FRET efficient. This feature is reflected in two parameters, the total number of groups per $\mathrm{QD}, n_{1,2}$, and the FRET parameter, $\gamma_{1,2}=\left(R_{\mathrm{o}} / r_{\mathrm{DA}}\right)^{6}{ }_{1,2}$ where $R_{\mathrm{o}}$ is the Förster distance and $r_{\mathrm{DA}}$ the donor-acceptor distance, one or both of which can vary between the two classes. The assumptions within the model are that the photochromic parameters are the same for both populations such that in absence of FRET the photostationary state for the two PC populations is $\sim 0.22$ upon exposure to UV (340 nm) irradiation. Ultraviolet light leads to photocyclization of oPC as well as photoreversion of $\mathrm{CPC}$ to oPC induced by direct excitation and indirectly via pcFRET ( $\mathrm{QD} \rightarrow \mathrm{cPC}$ ), whereas visible light induces photoreversion of cPC only by direct excitation.

Two coupled differential equations describe the time course of the oPC $\rightarrow \mathrm{cPC}$ transition in the two acceptor populations to the photostationary state upon exposure to UV light $\left(c_{1,2}[\mathrm{t}]=\right.$ $\left.\mathrm{cPC}[t] / \mathrm{QD} ; o_{1,2}[t]=\mathrm{oPC}[t] / \mathrm{QD} ; n_{1,2}=c_{1,2}[t]+o_{1,2}[t]\right):$

$$
\begin{aligned}
c_{1}^{\prime}[t]= & k_{\mathrm{oc}} Q_{\mathrm{oc}, \mathrm{uv}} \rho\left(n_{1}-c_{1}[t]\right)-k_{\mathrm{co}} Q_{\mathrm{co}, \mathrm{uv}} c_{1}[t] \\
& -k_{\mathrm{QD}} Q_{\mathrm{co}, \mathrm{vis}} E_{1}[t]
\end{aligned}
$$




$$
\begin{aligned}
c_{2}^{\prime}[t]= & k_{\mathrm{oc}} Q_{\mathrm{oc}, \mathrm{uv}} \rho\left(n_{2}-c_{2}[t]\right)-k_{\mathrm{co}} Q_{\mathrm{co}, \mathrm{uv}} c_{2}[t] \\
& -k_{\mathrm{QD}} Q_{\mathrm{co}, \mathrm{vis}} E_{2}[t] \\
E_{1}[t]= & \frac{c_{1}[t] \gamma_{1}}{1+c_{1}[t] \gamma_{1}+c_{2}[t] \gamma_{2}} ; \\
E_{2}[t]= & \frac{c_{2}[t] \gamma_{2}}{1+c_{1}[t] \gamma_{1}+c_{2}[t] \gamma_{2}} ; \quad n_{\mathrm{tot}}=n_{1}+n_{2} \\
Q_{\mathrm{co}, \mathrm{uv}}= & \rho\left(\frac{1-\alpha_{\mathrm{PS}}}{\alpha_{\mathrm{PS}}}\right)\left(\frac{\varepsilon_{\mathrm{oPC}, \mathrm{uv}}}{\varepsilon_{\mathrm{cPC}, \mathrm{uv}}}\right) Q_{\mathrm{oc}, \mathrm{uv}}
\end{aligned}
$$

quenching $\%[t]=100\left(E_{1}[t]+E_{2}[t]\right)$

in which the rate constants $k_{\mathrm{oc}} k_{\mathrm{co}}$, and $k_{\mathrm{QD}}$, for the forward and reverse photoreactions, and the QD-induced (via pcFRET) reverse reaction respectively, are products of the known irradiance and absorption cross sections. The corresponding quantum efficiency values $Q_{\text {oc,uv }}$ (for photoconversion of the antiparallel conformer), $Q_{\text {co,uv }}, Q_{c o, v i s}$, and $\gamma_{1}, \gamma_{2}, n_{1}$ and $n_{2}$ are fitted variables, two of which are dependent; $\rho$, the fraction of the photocyclization-competent antiparallel isomer of oPC, equals 0.40 according to NMR determinations. This value is maintained by dynamic equilibration with the unreactive parallel isomer during photoconversion. The FRET efficiencies $E_{1}$ and $E_{2}$ and thus the extent of QD quenching (eq 1) vary in time and are interdependent due to the different time courses of $c_{1}$ and $c_{2}$.

We simultaneously fit the photoconversion to $\operatorname{cPC}\left(c_{1,2}\right)$ and the $\mathrm{QD}$ quenching (quenching \%) as a function of the incremental UV irradiation dose using eq 1 and the FindMinimum routine of Mathematica (Wolfram Research). The fits are shown graphically in Figure 3 and Supporting Information S5 and the corresponding parameters are given in Table 1. The dual population model represents the data very well. Compared to the Gen-1 psQD, the new psQD has far fewer photochromic groups in total with only $\sim 3$ in class 1 , yet the FRET parameters $\gamma_{1,2}$ are 4-fold and 8 -fold greater, respectively, and the corresponding $\left(r_{\mathrm{DA}} / R_{\mathrm{o}}\right)_{1,2} 30 \%$ lower, resulting in a higher quenching efficiency in the photostationary state $(50 \%$ vs $40 \%)$. The larger value of $\gamma_{1}$ in the dual psQD probably reflects a reduction in $r_{\mathrm{DA}}$ of the class $1 \mathrm{PC}$, which due to the added linker were placed up to $8.5 \AA$ closer to the QD surface than in the Gen-1 psQD. The $R_{\mathrm{o}}$ computed for the QDcPC pair is $4.1 \mathrm{~nm}$ in both systems, such that if we ascribe the differences in $\gamma_{1}$ (see Table 1 ) to positional effects alone, class 1 acceptors would be located $8.3 \AA$ closer to the donor in the Gen-2 system, which is in excellent agreement with the stereochemical calculation. The linker may also result in better packing and orientation of the class $2 \mathrm{PC}$ within the polymer/ surface ligand matrix, accounting for the parallel reduction in $\gamma_{2}$.

The large absorption cross-section of the QD accounts for 84 and $75 \%$ of the $A_{340}$ in the open and photostationary PC states, respectively and leads to the condition $k_{\mathrm{QD}} \gg k_{\mathrm{oc}} k_{\mathrm{co}}$ reflected in the much faster equilibration (Figure $3 \mathrm{~B}$, inset) as well as in the displacement of the photostationary state to a very low steady-state value of $\alpha_{1, \mathrm{PS}}$. Interestingly, both $\alpha_{1, \mathrm{PS}}$ and $\alpha_{2, \mathrm{PS}}$ were similar for the two psQD generations. The derived quantum efficiency of photocyclization $\left(Q_{o c}, 0.4-0.5\right)$ significantly exceeds that for photoreversion $\left(Q_{c o, u v}, 0.1-0.2\right)$, which is also less efficient for excitation with visible light (or via

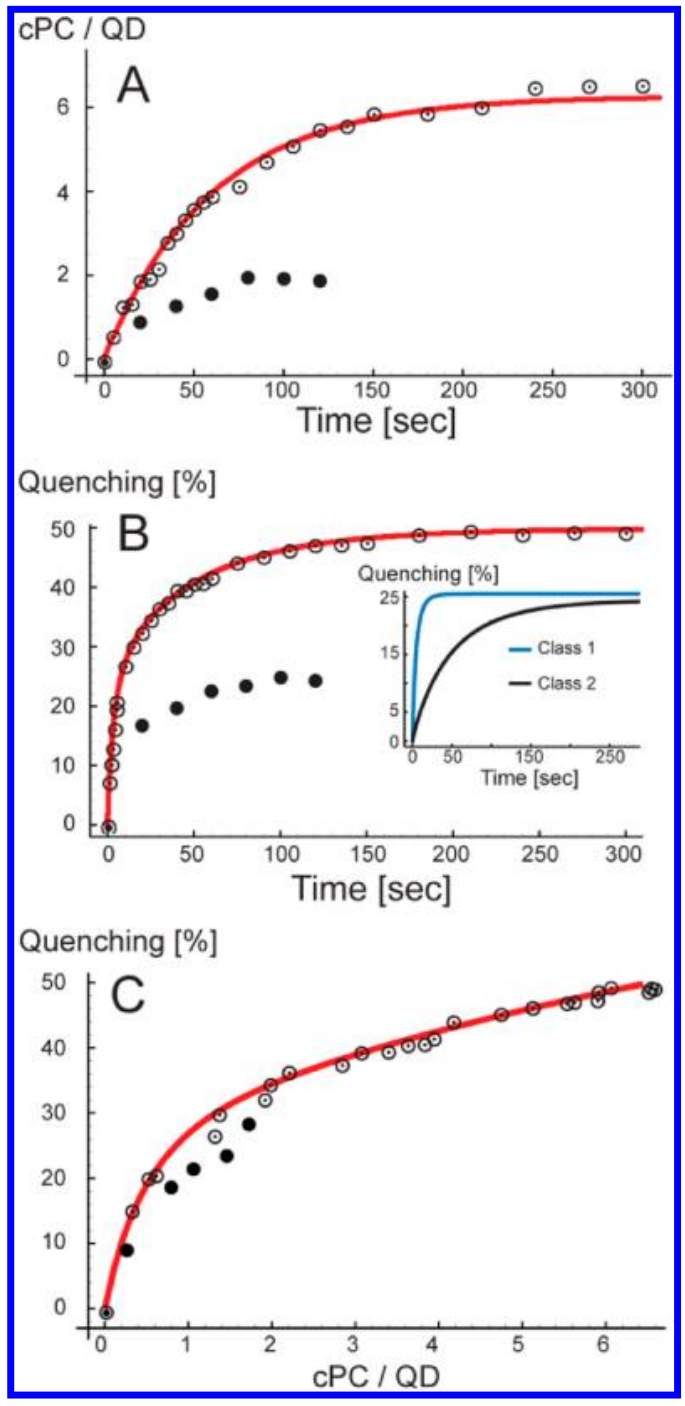

Figure 3. Photoconversion data and fits. Dual-color psQDs were irradiated with $340 \pm 10 \mathrm{~nm}$ UV light, irradiance $1.1 \mathrm{~mW} \mathrm{~cm}^{-2}[\odot$, Data]; [red line, Fit] or $365 \mathrm{~nm} \mathrm{UV} \mathrm{light,} \mathrm{irradiance} 1.05 \mathrm{~mW} \mathrm{~cm}^{-2}$ [๑, Data] for 1-15 s, absorbance and fluorescence spectra were recorded, and then a new pulse was applied. (A) Mean value of cPC molecules per $\mathrm{QD}$ as a function of irradiation time. (B) Fluorescence quenching of $\mathrm{QD}$ as a function of irradiation time. Inset: Quenching \% corresponding to the two classes of acceptor as a function of time: blue line, Class 1; black line, Class 2. (C) Fluorescence quenching of $\mathrm{QD}$ as a function of mean value of $\mathrm{cPC}$ molecules per $\mathrm{QD}$.

FRET) $\left(Q_{\text {co,vis }}, 0.01-0.02\right)$ compared to UV light. This result may be due to the intervention of additional, more efficient photoreversion pathways accessible to higher excited states in the latter case. ${ }^{44}$ The reverse cycloreversion reaction, induced by visible light $(546 \mathrm{~nm})$ and analyzed by corresponding adjustment of the physical constants and starting condition of eq 1 , yielded a slightly larger estimate for $Q_{c o, v i s}$ of 0.04 (Figure S5 in Supporting Information).

The above properties account for the nonintuitive phenomenon of very effective $\mathrm{QD}$ quenching despite the very low value of $\alpha_{1}$. That is, the system exhibits a "ping-pong" or "autoreset" property in the presence of $\geq 1$ class 1 or a larger number of class 2 cPC group(s). Excitation of the QD (favored under all conditions due to its very large absorption cross-section) is accompanied by a large probability of FRET transfer to the $\mathrm{cPC}$. As a consequence, light emission of the QD is aborted and 
Table 1. Analysis of the UV-Induced Photoconversion and QD Quenching Kinetics ${ }^{a}$

\begin{tabular}{|c|c|c|c|c|c|c|c|c|c|c|c|c|c|c|c|c|}
\hline $\mathrm{psQD}$ & $Q_{o c}$ & $Q_{\mathrm{couvv}}$ & $Q_{c o, v i s}$ & $n_{1}$ & $n_{2}$ & $\gamma_{1}$ & $\gamma_{2}$ & $k_{\mathrm{oc}}$ & $k_{\mathrm{co}}$ & $k_{\mathrm{QD}}$ & $E_{1, \mathrm{PS}}$ & $E_{2, \mathrm{PS}}$ & $\alpha_{1, \mathrm{PS}}$ & $\alpha_{2, \mathrm{PS}}$ & $\left(r_{\mathrm{DA}} / R_{\mathrm{o}}\right)_{1}$ & $\left(r_{\mathrm{DA}} / R_{\mathrm{o}}\right)_{2}$ \\
\hline \multirow[t]{2}{*}{ Gen-2 } & 0.44 & 0.14 & 0.015 & 3 & 32 & 6.6 & 0.08 & 0.01 & 0.08 & 2.2 & 0.26 & 0.24 & 0.02 & 0.20 & 0.7 & 1.5 \\
\hline & 0.6 & 3.0 & 1.4 & 1.2 & 1.6 & 4.8 & 2.0 & & & & & & & & & \\
\hline Gen-1 & 0.52 & 0.17 & 0.015 & 14 & 81 & 1.5 & 0.01 & 0.01 & 0.08 & 0.8 & 0.32 & 0.03 & 0.03 & 0.18 & 0.9 & 2.1 \\
\hline
\end{tabular}

${ }^{a}$ Gen-2, this study; Gen-1, PMA 6 PC 70 C12, from ref 22. Values in italics, relative deviations in \% beyond which the increase in SSQ (sum of squares) of the fitting routine exceeds $10 \%$. The units of $k_{\mathrm{oc}} k_{\mathrm{co}}$, and $k_{\mathrm{QD}}$ are $\mathrm{s}^{-1}$.

the acceptor PC reverts to the open state with fairly high efficiency.

Figure 3 also features data for UV photoconversion at 365 $\mathrm{nm}$, a more accessible wavelength for fluorescence microscopy. Because of the less favorable oPC/cPC absorption cross-section ratio, the photostationary state was displaced to lower values of $\alpha_{\mathrm{PS}}$, and $\mathrm{QD}$ quenching diminished in accordance with the universal quantitative relation between quenching and $\mathrm{cPC} /$ QD content, established by exposure to $340 \mathrm{~nm}$ (Figure 3C).

We measured the fluorescence decay kinetics of the dualcolor psQD in different states to confirm that the observed photomodulation was due to FRET. The decays of the parent QD and psQD were complex (Figure 4), requiring four

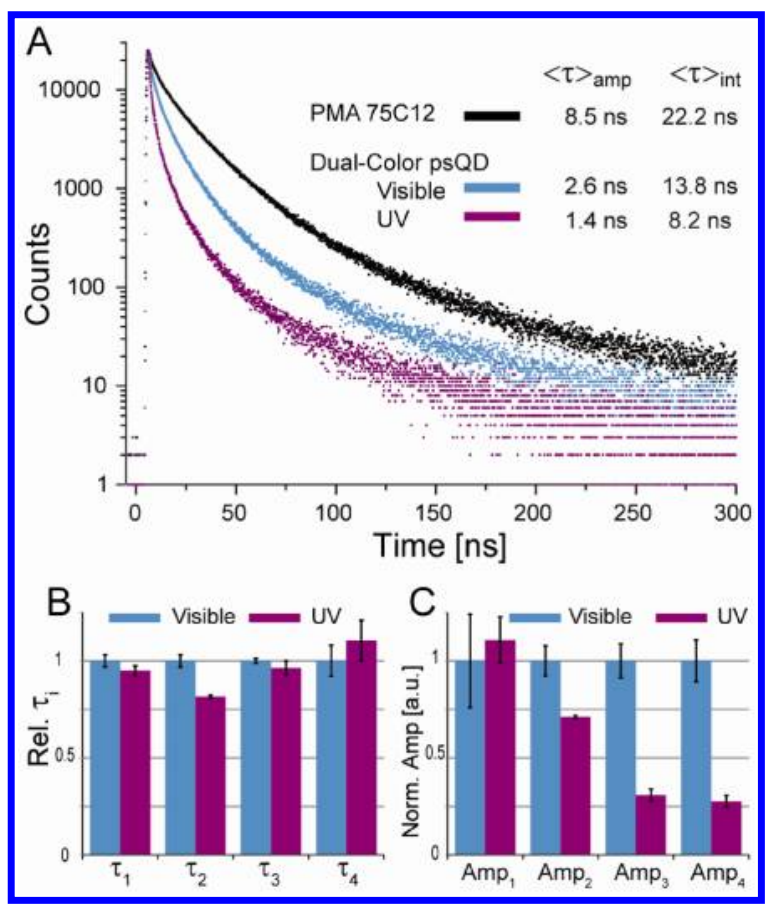

Figure 4. (A) Fluorescence-detected excited-state decays determined at $550 \mathrm{~nm}$. (B) Individual decay constants normalized by the values for the oPC state. Absolute decay constants: oPC (initial state) $\tau_{1-4}$, $[0.48 ; 3.8 ; 12.7 ; 60 \mathrm{~ns}]$; photostationary state $\tau_{1-4}$, [0.45; 3.12; 12.2; $66 \mathrm{~ns}$ ]. (C) Individual amplitudes, normalized by the oPC values. The amplitudes were converted to a common absolute scale by division by $\Sigma \mathrm{amp}_{i, \text { counts }} \cdot \tau_{i}$ and multiplication by the steady-state spectrafluorimeter intensities. Relative amplitudes for the oPC state, $\mathrm{amp}_{1-4},[1 ; 0.25$; $0.10 ; 0.007]$ and for photostationary state; $\mathrm{amp}_{1-4},[1 ; 0.17 ; 0.03$; $0.002]$.

exponential components for the QD (and two for the Alexa647) to adequately represent the time course of excited state deactivation. In such Type II QDs, the exciton hole is confined to the core while the electron is delocalized in the core and first shell. ${ }^{42}$ The outer liganded passivation shell accounts for a high quantum yield of core-shell-shell QDs. ${ }^{35}$ The lower value of the final psQD indicates the presence of surface traps equilibrating with the radiative process of hole-electron recombination and/or the presence of defects and ligands contributing to nonradiative pathways. ${ }^{45}$

The psQD time-resolved decays of samples undergoing photoconversion cycles are best interpreted by examining the individual lifetimes and amplitudes (Figure 4), as well as two derived global means: amplitude weighed lifetime, $\langle\tau\rangle_{\mathrm{amp}}$ and intensity weighed lifetime, $\langle\tau\rangle_{\text {int }}$ (Table 2). Analysis by an

Table 2. Amplitude Weighed Mean Lifetime $\langle\tau\rangle_{\mathrm{amp}}$ and Intensity Weighed Mean Lifetime $\langle\tau\rangle_{\text {int }}$ of the Dual-Color psQD in the Two Photostationary States, and of Controls, Measured on a FluoroLog (Horiba Jobin Yvon) TCSPC System (Lifetime Values in ns)

$\begin{array}{cccc}\begin{array}{c}\text { QD } \\ \text { detection }^{a}\end{array} & \begin{array}{c}\text { polymer } \\ \text { coated }^{c}\end{array} & \begin{array}{c}\text { dual-color psQD } \\ \text { oPC (Vis) }\end{array} & \begin{array}{c}\text { dual-color psQD } \\ \text { oPC, cPC (UV) }\end{array} \\ \langle\tau\rangle_{\text {amp }} & 8.5 \pm 0.1 & 2.6 \pm 0.5 & 1.4 \pm 0.1 \\ \langle\tau\rangle_{\text {int }} & 22.2 \pm 0.2 & 13.8 \pm 0.2 & 8.2 \pm 0.2 \\ \begin{array}{c}\text { Alexa647 } \\ \text { detection }^{b}\end{array} & \text { in solution } & \begin{array}{c}\text { dual-color psQD } \\ \text { oPC (Vis) }\end{array} & \begin{array}{c}\text { dual-color psQD } \\ \text { oPC, cPC (UV) }\end{array} \\ \langle\tau\rangle_{\text {amp }} & 1.28 \pm 0.01 & 1.18 \pm 0.01 & 1.14 \pm 0.01 \\ \langle\tau\rangle_{\text {int }} & 1.17 \pm 0.06 & 1.06 \pm 0.05 & 1.02 \pm 0.02\end{array}$

${ }^{a}$ Excitation with nanoLED N-320 nm and detection at $550 \mathrm{~nm}$. ${ }^{b}$ Excitation with nanoLED N-560 nm and detection at $660 \mathrm{~nm}$. ${ }^{c}$ CANdot CSS $540 \mathrm{~nm}$ QDs coated with amphiphilic polymer lacking PC and Alexa probe. ${ }^{d}$ Photostationary state (oPC and cPC equilibrium) established by UV irradiation.

extended exponential $\left(\exp \left[-\left(t / \tau_{\text {ext }}\right)^{\beta}\right]\right)^{41,45}$ was also carried out but did not provide additional insights. FRET systems with donors presenting multiple decays are generally represented in terms of $\langle\tau\rangle_{\text {amp }}$ since for a given impulse response amplitude this quantity reflects the total emission under the decay curve. $^{46,47}$ In fact, $\langle\tau\rangle_{\mathrm{amp}}$ diminished by $47 \pm 3 \%(2.6 \rightarrow 1.4 \mathrm{~ns})$ in three successive oPC $\rightarrow \mathrm{cPC}$ transitions, a value identical to that obtained from the steady-state emission and also indicative of a lack of undetected dark states. ${ }^{46}$ The corresponding change in $\langle\tau\rangle_{\text {int }}$ was smaller $(40 \%)$. The individual four decay constants $\left(\tau_{1-4}: \sim 0.5,4,13,60 \mathrm{~ns}\right)^{48}$ did not vary systematically during photocycling but the amplitudes, amp $\mathrm{p}_{2,3,4}$ strongly reflected the global quenching, decreasing by 30,71 , and $68 \%$, respectively, upon photocyclization (Figure 4B,C). On the basis of the decay intensities, components 2 and 3 were the major contributors to the QD emission.

There was a significant decrease in the $\langle\tau\rangle_{\mathrm{amp}}$ of QDs coated with an unmodified amphiphilic polymer upon passing to the dual-color psQD system. Previous investigations ${ }^{49}$ have rationalized this effect in terms of FRET and/or screening processes but we propose that the coating efficiency plays a larger role in our system. A polymer only containing pendant alkyl chains will coat more efficiently than one carrying voluminous dyes such as PC and Alexa647. The Alexa647 probe exhibited a slightly shorter lifetime when conjugated to 
psQD than in solution but the sensitivity to PC photocyling was negligible.

In conclusion, we have prepared a water-soluble dual-color nanoparticle with ratiometric imaging capabilities. The technique is based on coating a QD with a dye-doped amphiphilic photochromic polymer providing solubility in aqueous media. The dual-color $p s Q D$ are distinguished by small size (5.5 nm diameter with a $14 \mathrm{~nm}$ hydrodynamic diameter), biocompatibility, a broad excitation spectra, dual emission at 550 and $666 \mathrm{~nm}$, a tunable emission ratio with up to $100 \%$ change upon photochromic switching, and distinct fluorescence lifetimes for the different components and states. A very advantageous feature of the $\mathrm{psQD}$ system is the prominent quenching of the $\mathrm{QD}$ exerted by low degrees of photoconversion. This effect, attributable to pcFRET as indicated above, should greatly facilitate achieving the modulation required for sensitive lock-in detection. ${ }^{31}$

The properties of the dual-color psQD should lead to manifold applications in cellular imaging, particular due to the greater sensitivity, selectivity, and background rejection afforded by the dual emission and photoswitchable ratiometric signals. We envision the potential of using laser light sources for precise control of the ER of single nanoparticles, as in singleparticle tracking. Numerous combinations of dyes, sensors, and nanoparticles are feasible since the polymer preparation can be fine-tuned to obtain different functionalities as well as improving existing ones. For example, the addition of a linker to the PC group placed the FRET acceptor closer to the QD, such that the photochromic quenching of the psQD increased by $30 \%$ while the number of PC groups required decreased by $60 \%$ in comparison to the Gen-1 constructs. Because of their decay kinetics, the psQDs are also ideal for FLIM. Additional PC tailoring such as improved photoconversion and red-shifted spectra could further improve the utility of the nanoparticles in the cellular context, for example, in imaging strategies for pulsechase experiments based on spatiotemporally structured illumination. The psQD probes may also be suited for applications in super-resolution microcopy. ${ }^{5,50}$

\section{ASSOCIATED CONTENT}

\section{S Supporting Information}

Synthesis and characterization of PCahx; TEM images; details regarding the photophysical and computational data. This material is available free of charge via the Internet at http:// pubs.acs.org.

\section{AUTHOR INFORMATION}

\section{Corresponding Author}

*E-mail: tjovin@gwdg.de.

\section{Notes}

The authors declare no competing financial interest.

\section{ACKNOWLEDGMENTS}

Elizabeth A. Jares-Erijman, the corresponding author and thesis supervisor of S.A.D., passed away during the final revisions of the manuscript. The surviving authors dedicate this publication to her memory and in acknowledgment of her inspiration, leadership, contributions, and outstanding personal qualities. We thank D. Arndt-Jovin and M. Konrad for valuable discussions, D. Riedel and G. Heim for the TEM images, NMR-Service, Georg-August-Universität (Göttingen, Germany) for NMR spectra, and the Department of Physical
Biochemistry, MPIbpc, for the use of the TCSPC fluorimeter. This study was supported by the Max Planck Society (E J.-E., Partner Group grant; T.M.J and E.J.-E., Toxic Protein Conformation project), Argentine agencies ANPCyT, CONICET, UBA (E.J.-E.), and Cluster of Excellence 171 of the DFG Centre for the Molecular Physiology of the Brain (DFG CMPB). S.A.D was supported by the Deutscher Akademischer Austauschdienst (DAAD-Sandwich Doctorate Scholarship No. A/09/75106).

\section{REFERENCES}

(1) Fu, A.; Gu, W.; Larabell, C.; Alivisatos, A. P. Curr. Opin. Neurobiol. 2005, 15, 568-575.

(2) Delehanty, J. B.; Bradburne, C. E.; Susumu, K.; Boeneman, K.; Mei, B. C.; Farrell, D.; Blanco-Canosa, J. B.; Dawson, P. E.; Mattoussi, H.; Medintz, I. L. J. Am. Chem. Soc. 2011, 133, 10482-10489.

(3) Bruchez, M. P. Curr. Opin. Chem. Biol. 2011, 15, 775-780.

(4) Pinaud, F.; Clarke, S.; Sittner, A.; Dahan, M. Nat. Methods 2010, 7, 275-285.

(5) Leung, B. O.; Chou, K. C. Appl. Spectrosc. 2011, 65, 967-980.

(6) Yu, W. W. Expert Opin. Biol. Ther. 2008, 8, 1571-1581.

(7) Sperling, R. A.; Parak, W. J. Philos. Trans. R. Soc. London, Ser. A 2010, 368, 1333-1383.

(8) Zhang, F.; Ali, Z.; Amin, F.; Riedinger, A.; Parak, W. J. Anal. Bioanal. Chem. 2010, 397, 935-942.

(9) Erno, Z.; Yildiz, I.; Gorodetsky, B.; Raymo, F. M.; Branda, N. R. Photochem. Photobiol. Sci. 2010, 9, 249-253.

(10) Yildiz, I.; Tomasulo, M.; Raymo, F. M. J. Mater. Chem. 2008, 18, $5577-5584$.

(11) Medintz, I. L.; Trammell, S. A.; Mattoussi, H.; Mauro, J. M. J. Am. Chem. Soc. 2004, 126, 30-31.

(12) Rogach, A. L.; Klar, T. A.; Lupton, J. M.; Meijerink, A.; Feldmann, J. J. Mater. Chem. 2009, 19, 1208-1221.

(13) Jares-Erijman, E. A.; Jovin, T. M. Nat. Biotechnol. 2003, 21, $1387-1395$

(14) Giordano, L.; Jovin, T. M.; Irie, M.; Jares-Erijman, E. A. J. Am. Chem. Soc. 2002, 124, 7481-7489.

(15) Jares-Erijman, E.; Giordano, L.; Spagnuolo, C.; Lidke, K.; Jovin, T. Mol. Cryst. Liq. Cryst. 2005, 430, 257-265.

(16) Giordano, L.; Vermeij, R. J.; Jares-Erijman, E. A. Arkivoc 2005, 268-281.

(17) Irie, M. Chem. Rev. 2000, 100, 1685-1716.

(18) Irie, M. Photochem. Photobiol. Sci. 2010, 9, 1535-1542.

(19) Polyakova, S. M.; Belov, V. N.; Bossi, M. L.; Hell, S. W. Eur. J. Org. Chem. 2011, 2011, 3301-3312.

(20) Hirose, T.; Matsuda, K.; Irie, M. J. Org. Chem. 2006, 71, 74997508.

(21) Saitoh, M.; Fukaminato, T.; Irie, M. J. Photochem. Photobiol., A 2009, 207, 28-31.

(22) Díaz, S. A.; Menendez, G. O.; Etchehon, M. H.; Giordano, L.; Jovin, T. M.; Jares-Erijman, E. A. ACS Nano 2011, 5, 2795-2805.

(23) Pellegrino, T.; Manna, L.; Kudera, S.; Liedl, T.; Koktysh, D.; Rogach, A. L.; Keller, S.; Rädler, J.; Natile, G.; Parak, W. J. Nano Lett. 2004, 4, 703-707.

(24) Díaz, S. A.; Toscani, A. M.; Arndt-Jovin, D. J.; Jovin, T. M.; Jares-Erijman, E. A. Small Photostable Photoswitchable Quantum Dots as Nanotools for Live Cell Imaging. In Nanotech; NSTI, Ed.; CRC Press: Boston, USA, 2011; Vol. 3, pp 205-208.

(25) Zhang, F.; Lees, E.; Amin, F.; RiveraGil, P.; Yang, F.; Mulvaney, P.; Parak, W. J. Small 2011, 7, 3113-3127.

(26) Ruan, G.; Winter, J. O. Nano Lett. 2011, 11, 941-945.

(27) Zhu, L.; Wu, W.; Zhu, M.-Q.; Han, J. J.; Hurst, J. K.; Li, A. D. Q. J. Am. Chem. Soc. 2007, 129, 3524-3526.

(28) Chen, J.; Zhang, P.; Fang, G.; Yi, P.; Yu, X.; Li, X.; Zeng, F.; Wu, S. J. Phys. Chem. B 2011, 115, 3354-3362.

(29) Genovese, D.; Montalti, M.; Prodi, L.; Rampazzo, E.; Zaccheroni, N.; Tosic, O.; Altenhöner, K.; May, F.; Mattay, J. Chem. Commun. 2011, 47, 10975-10977. 
(30) Osakada, Y.; Hanson, L.; Cui, B. Chem. Commun. 2012, 48, 3285-3287.

(31) Marriott, G.; Mao, S.; Sakata, T.; Ran, J.; Jackson, D. K.; Petchprayoon, C.; Gomez, T. J.; Warp, E.; Tulyathan, O.; Aaron, H. L.; Isacoff, E. Y.; Yan, Y. Proc. Natl. Acad. Sci. U.S.A. 2008, 105, 1778917794.

(32) Colyer, R. A.; Lee, C.; Gratton, E. Microsc. Res. Techniq. 2008, 71, 201-213.

(33) Fernández-Argüelles, M. T.; Yakovlev, A.; Sperling, R. A.; Luccardini, C.; Gaillard, S.; Sanz Medel, A.; Mallet, J.-M.; Brochon, J.C.; Feltz, A.; Oheim, M.; Parak, W. J. Nano Lett. 2007, 7, 2613-2617.

(34) Janczewski, D.; Tomczak, N.; Han, M.-Y.; Vancso, G. J. Nat. Protoc. 2011, 6, 1546-1553.

(35) Talapin, D. V.; Mekis, I.; Götzinger, S.; Kornowski, A.; Benson, O.; Weller, H. J. Phys. Chem. B 2004, 108, 18826-18831.

(36) Parak, W. J.; Pellegrino, T.; Plank, C. Nanotechnology 2005, 16, R9-R25.

(37) Clapp, A. R.; Medintz, I. L.; Mattoussi, H. ChemPhysChem 2006, 7, 47-57.

(38) Sadhu, S.; Patra, A. ChemPhysChem 2008, 9, 2052-2058.

(39) Algar, W. R.; Wegner, D.; Huston, A. L.; Blanco-Canosa, J. B.; Stewart, M. H.; Armstrong, A.; Dawson, P. E.; Hildebrandt, N.; Medintz, I. L. J. Am. Chem. Soc. 2012, 134, 1876-1891.

(40) Levitus, M.; Ranjit, S. Q. Rev. Biophys. 2010, 44, 123-151.

(41) Cooper, D. R.; Suffern, D.; Carlini, L.; Clarke, S. J.; Parbhoo, R.; Bradforth, S. E.; Nadeau, J. L. Phys. Chem. Chem. Phys. 2009, 11, 4298-4310.

(42) Carrillo-Carrion, C.; Cardenas, S.; Simonet, B. M.; Valcarcel, M. Chem. Commun. 2009, 5214-5226.

(43) Llopis, M. V.; Rodríguez, J. C. C.; Martín, F. J. F.; Coto, A. M.; Fernández-Argüelles, M. T.; Costa-Fernández, J. M.; Sanz-Medel, A. Nanotechnology 2011, 22, 385703.

(44) Murakami, M.; Miyasaka, H.; Okada, T.; Kobatake, S.; Irie, M. J. Am. Chem. Soc. 2004, 126, 14764-14772.

(45) Schlegel, G.; Bohnenberger, J.; Potapova, I.; Mews, A. Phys. Rev. Lett. 2002, 88, 137401.

(46) Sillen, A.; Engelborghs, Y. Photochem. Photobiol. 1998, 67, 475486.

(47) Wu, P. G.; Brand, L. Anal. Biochem. 1994, 218, 1-13.

(48) "Lifetimes" are inadequate terms for the reciprocal eigenvalues corresponding to excited state dynamics because they generally do not reflect the deactivation of distinct chemical species but rather of a network of excited states; the observed "amplitudes" are the eigenvectors.

(49) Niebling, T.; Zhang, F.; Ali, Z.; Parak, W. J.; Heimbrodt, W. J. Appl. Phys. 2009, 106, 104701.

(50) Dertinger, T.; Colyer, R.; Iyer, G.; Weiss, S.; Enderlein, J. Proc. Natl. Acad. Sci. U.S.A. 2009, 106, 22287-22292. 